\title{
In Situ TEM Observation of Water Splitting
}

Julio A. Rodríguez Manzo ${ }^{1}$, Norman J. Salmon ${ }^{1}$ and Daan Hein Alsem ${ }^{1}$.

1. Hummingbird Scientific, Lacey, USA.

Now it is possible to perform in situ/operando liquid electrochemistry TEM experiments with the help of dedicated TEM sample holders [1]. These studies of electrochemical reactions provide, foremost, high spatial resolution maps of the site where the reaction occurs. Additionally, electron diffraction and spectroscopy analysis can tell us about reaction-induced structural and chemical changes in specific materials of the studied system. Overall electrochemical states can be concurrently monitored with standard techniques such as voltammetry, which is used to analyze reduction-oxidation reactions by correlating changes in current with chemical transformations.

The challenge of controlling how water is split into its constituents-gaseous hydrogen and oxygenwhen an electrical current is passed through it has broad technological implications in devices that use hydrogen-based energy storage strategies. In this context, we describe an experimental setup used to split water within a TEM for in situ observations and show an example where we correlate TEM images of gas bubble formation - a hallmark of water splitting - at a biased metal electrode immersed in an electrolyte with the corresponding voltammetry analysis. The description of the experimental setup includes details of the in situ liquid TEM sample holder (Figure 1), MEMS-based electrochemistry cell chips with electron-transparent windows, basic circuitry, and imaging conditions.

All observations were made with a JEOL JEM-2100 LaB 6 TEM operated at $200 \mathrm{kV}$; image recording (Direct Electron DE-12 camera) was done with acquisition rates of 20-30 fps; and voltammetry measurements were done with a potentiostat equipped with a low current amplifier with noise levels < pA (BioLogic SP-200).

Results of a typical in situ water splitting TEM experiment are shown in Figure 2, where we show the dynamics of gas bubble production at a metal electrode immersed in an electrolyte (water plus $\mathrm{NaCl}$ ) at corresponding potentials from the voltammetry measurement. The TEM images highlight this approach's advantages: we can pinpoint exactly where the bubbles originate, their size and shape, and how they travel away from the electrode-all concurrent with voltammetry data that informs about the chemical state at different potentials at different times. Of course, the electron beam's effect on the reaction must be considered when interpreting these results [2].

We think in situ water splitting TEM studies will help to close information gaps, particularly in the areas of elucidating reaction sites by improving spatial resolution, and establishing the structural and chemical states of electrodes during the reaction.

\section{References:}

[1] Frances M. Ross in "Liquid cell electron microscopy", ed. Frances M. Ross, (Cambridge University Press, New York City) p.191.

[2] Nicholas M. Schneider et al, The Journal of Physical Chemistry C 118 (2014), p. 22373. 


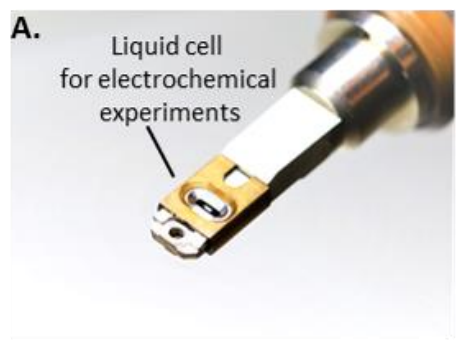

B.

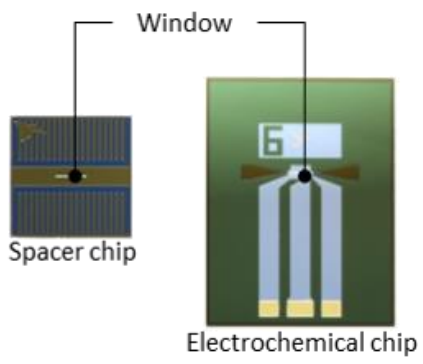

Figure 1. Details of closed-cell liquid flow TEM sample holder tip (A) and MEMS-based electrochemistry cell chips with electron-transparent windows (B) used to perform water splitting experiments.
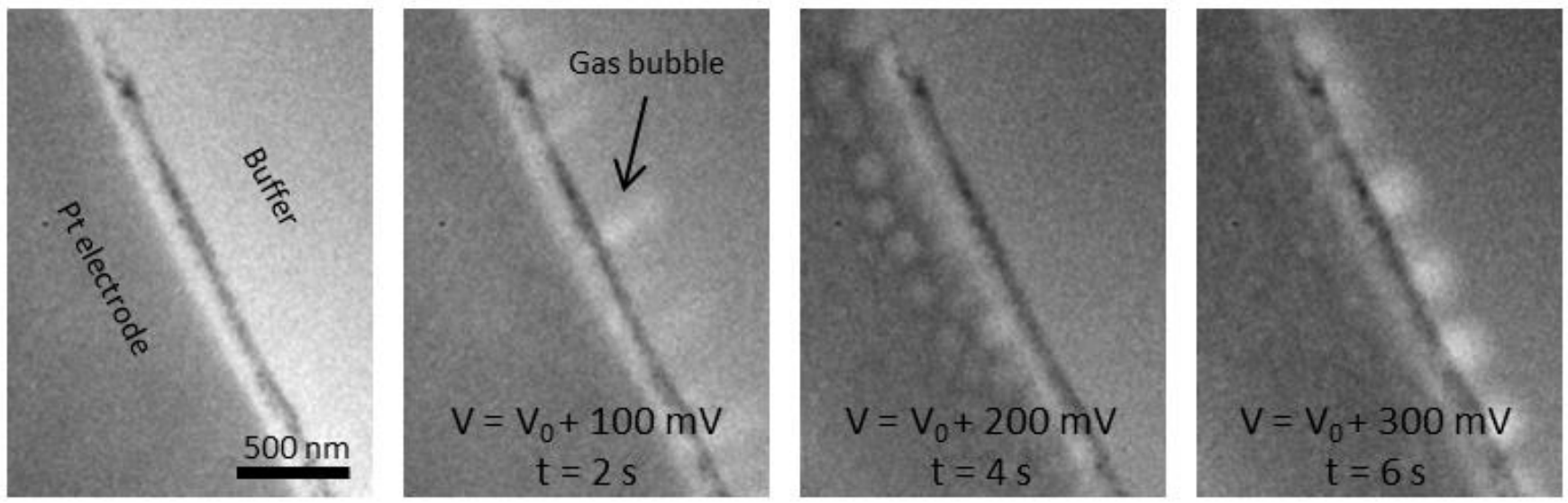

Figure 2. Gas bubble production at a metal electrode immersed in an electrolyte. From left to right, the sequence of TEM images shows when and where gas bubbles - lighter regions - are produced at the metal electrode as its potential varies. $\mathrm{V}, \mathrm{V}_{0}$, and $\mathrm{t}$ indicate potential, reference potential and time, respectively. 